\title{
CHARGE SOLITONS IN 1-D ARRAY OF MESOSCOPIC TUNNEL JUNCTIONS
}

\author{
M. AMMAN ${ }^{a, b}$, E. BEN-JACOB ${ }^{\text {a,b,c }}$ and K. MULLEN ${ }^{a}$ \\ - Department of Physics, The University of Michigan, Ann Arbor, MI 48109, USA \\ - Scientific Laboratory, Ford Motor Company, Dearborn, MI 48121, USA \\ c School of Physics and Astronomy, Raymond and Beverly Sackler Faculty of Exact Sciences, Tel-Aviv University, \\ 69978 Tel-Aviv, Israel
}

Received 18 August 1989; accepted for publication 12 October 1989

Communicated by A.A. Maradudin

\begin{abstract}
We study the dynamics of a one-dimensional array of mesoscopic normal tunnel junctions using the semiclassical picture. We show that the charging effect on a single electron tunneling through a single junction together with capacitive coupling to a substrate lead to the existence of soliton-like modes of charge transfer along the array. These modes are manifested by the appearance of clear Coulomb steps in the current-voltage characteristics (for a range of junction parameters) which can be directly verified experimentally.
\end{abstract}

Charging effects can lead to temporal correlations in the transfer of charge through current-driven mesoscopic normal tunnel junctions $[1,2]$. The voltage across such junctions oscillates in time at a frequency of $I / e$, where $I$ is the external current. The analogy of these oscillations with the Josephson effect naturally suggests the possibility for the existence of soliton-like charge propagation along a one-dimensional array of coupled mesoscopic normal tunnel junctions [3-6] (analogous to a long Josephson junction). We consider a set of serially coupled junctions, as shown in fig. 1 . The interjunction region is capacitively coupled to a substrate with capacitance $C_{\mathrm{s}}$ which, as we will see, has a very crucial role in the dynamics of the system. The charge on $C_{\mathrm{s}}$ equals the difference in the charge between the two adjacent junctions, so that $C_{\mathrm{s}}$ controls the value of the coupling along the chain.

In a previous publication [5] we have predicted the existence and studied the dynamics of solitons when the system is uniformly driven, that is, applying the same voltage to each segment of the array. We also considered only the simple limit of "local" charging energy in which the charging energy of each junction depended on the capacitance of that specific junction. Here, in addition, we also consider the limit of "nonlocal" charging energy. In this limit, the charging energy depends on the redistribution of the voltages across all the junctions after a charge has transferred across a given junction. We study here an asymmetrically driven system [6] as shown in fig. 1, which is somewhat easier for experimental realization. To study the dynamics of the array we assume the semiclassical picture to describe the dynamics of each of the individual junctions. We also assume

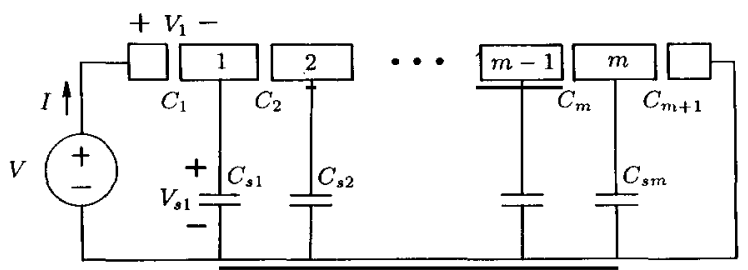

Fig. 1. Schematic representation of a mesoscopic tunnel junction array formed from $m$ number of submicron metal droplets. The droplets are coupled to each other such that tunneling can occur between the adjacent droplets. The droplets are also capacitively coupled to a substrate, but no tunneling is allowed between the droplets and the substrate. The entire system is driven at one end by the voltage source $V$. 
that the charge relaxation time of the interjunction regions are much faster than the time of tunneling $[7,8]$ and the time between tunneling events. Otherwise there is an additional inertia term as described in ref. [5]. All calculations were done at zero temperature for arrays of identical junctions.

The main results are: the overall $I-V$ characteristics for most of the parameters are very similar to the $I-V$ characteristic of a current-driven single junction as was found in refs. $[9,10]$. In the intermediate and small coupling limit ( $C_{\mathrm{s}}$ not much smaller than the junctions' capacitance) there are soliton-like modes of propagation of charge along the array. These modes exist at very low currents for which the $I-V$ characteristic is nonlinear. They can be observed directly by either probing the voltage across a single junction or by measuring the time dependence of the current. They are also manifested as clear steps in the $I-V$ characteristics.

The one-dimensional array of mesoscopic tunnel junctions is composed of $m$ metal electrodes (droplet or interjunction regions) coupled to each other so that tunneling can occur between adjacent droplets, thus forming $m+1$ tunnel junctions (fig. 1). The droplets are also capacitively coupled to a substrate, but we exclude tunneling between the droplets and the substrate. Here we consider the system to be driven at one end by an ideal voltage source $V$.

We use the semiclassical model to describe the junction array $[2,11]$. In this model the state of each junction is described by the voltage dropped across the junction, a classical variable. The state of the system is then described by all the junction voltages, $\left\{V_{j}\right\}$. The tunneling of an electron on a junction will change the charge distribution and thus every junction voltage. It is convenient to describe the state of the system by $\left\{N_{j}\right\}$, the number of surplus electrons contained on each droplet. In order to relate $\left\{N_{j}\right\}$ and $\left\{V_{j}\right\}$ we sum to zero the voltages around closed loops in the circuit of fig. 1 and obtain the following equations:

$$
\begin{aligned}
& \sum_{i=1}^{m+1} V_{i}-V=0, \\
& -V_{\mathrm{s} i}+V_{\mathrm{i}+1}+V_{\mathrm{s} i+1}=0, \quad 1 \leqslant i \leqslant m-1, \\
& -V_{\mathrm{s} m}+V_{m+1}=0,
\end{aligned}
$$

where $V_{s i}$ is the voltage difference between the $i$ th droplet and the substrate. We also sum the charge on each droplet and set this sum equal to $-N_{i} e$, the extra charge due to electron tunneling. The resulting equations are

$-V_{i} C_{i}+V_{\mathrm{s} i} C_{\mathrm{s} i}+V_{i+1} C_{i+1}=-N_{i} e, \quad 1 \leqslant i \leqslant m$,

where $C_{i}$ is the capacitance of the $i$ th junction and $C_{s i}$ is the capacitance coupling the $i$ th droplet to the substrate. In matrix form the equations are represented by

$$
\left(\begin{array}{ccccccccccc}
1 & 1 & 1 & 1 & \ldots & 1 & 0 & 0 & 0 & \ldots & 0 \\
C_{1} & -C_{2} & 0 & 0 & \ldots & 0 & -C_{\mathrm{s} 1} & 0 & 0 & \ldots & 0 \\
0 & C_{2} & -C_{3} & 0 & \ldots & 0 & & -C_{\mathrm{s} 2} & 0 & \ldots & 0 \\
\vdots & & & & & & 0 & & & & \\
0 & 0 & 0 & \ldots & C_{m} & -C_{m+1} & 1 & 0 & 0 & \ldots & -C_{\mathrm{s} m} \\
0 & 1 & 0 & 0 & \ldots & 0 & -1 & 1 & 0 & \ldots & 0 \\
0 & 0 & 1 & 0 & \ldots & 0 & 0 & -1 & 1 & \ldots & 0 \\
\vdots & & & & & & \vdots & & & & \\
0 & 0 & 0 & \ldots & 0 & 1 & 0 & 0 & \ldots & 0 & -1
\end{array}\right)\left(\begin{array}{c}
V_{1} \\
V_{2} \\
V_{3} \\
\vdots \\
V_{m+1} \\
V_{\mathrm{s} 1} \\
V_{\mathrm{s} 2} \\
\vdots \\
V_{\mathrm{s} m}
\end{array}\right)=\left(\begin{array}{c}
V \\
N_{1} e \\
N_{2} e \\
N_{3} e \\
\vdots \\
N_{m} e \\
0 \\
0 \\
\vdots \\
0
\end{array}\right) .
$$

From these equations, the voltage dropped across each junction can be determined given $\left\{N_{j}\right\}$ and $V$. Alternatively, a general expression for $V_{i}$ can be determined by solving a difference equation formed from (2) and (4) subject to the constraints of (1) and (3) [12]. 
The time evolution of the array is determined by a stochastic process composed of single electron tunneling events that alter $\left\{N_{j}\right\}$. This stochastic process is given by

$$
\begin{aligned}
\left\{N_{j}(t+\Delta t)\right\} & =\left\{N_{j}(t), N_{1}(t)+1\right\}_{j \neq 1}, & & \text { with probability } l_{1}\left(V_{1}\right) \Delta t, \\
& =\left\{N_{j}(t), N_{1}(t)-1\right\}_{j \neq 1}, & & \text { with probability } r_{1}\left(V_{1}\right) \Delta t, \\
& =\left\{N_{j}(t), N_{1}(t)-1, N_{2}(t)+1\right\}_{j \neq 1,2}, & & \text { with probability } l_{2}\left(V_{2}\right) \Delta t, \\
& =\left\{N_{j}(t), N_{1}(t)+1, N_{2}(t)-1\right\}_{j \neq 1,2}, & & \text { with probability } r_{2}\left(V_{2}\right) \Delta t, \\
& \ldots & & \\
& =\left\{N_{j}(t), N_{i-1}(t)-1, N_{i}(t)+1\right\}_{j \neq i-1, i}, & & \text { with probability } l_{i}\left(V_{i}\right) \Delta t, \\
& =\left\{N_{j}(t), N_{i-1}(t)+1, N_{i}(t)-1\right\}_{j \neq i-1, i}, & & \text { with probability } r_{i}\left(V_{i}\right) \Delta t, \\
& \cdots & & \\
& =\left\{N_{j}(t)\right\}, & & \text { with probability } 1-\sum_{i=1}^{m+1}\left[r_{i}\left(V_{i}\right)+l_{i}\left(V_{i}\right)\right] \Delta t,
\end{aligned}
$$

where $r_{i}\left(V_{i}\right)$ and $l_{i}\left(V_{i}\right)$ are the instantaneous rates for electron tunneling from the right and left on the $i$ th junction.

The electron tunneling rates are determined from a "Golden Rule" calculation and, at zero temperature, are given by [11]

$$
\begin{aligned}
r_{i}\left(V_{i}\right) & =\Delta \mu_{i} / e^{2} R_{i}, & & \Delta u_{i}>0, \\
& =0, & & \text { otherwise, }
\end{aligned}
$$

where $R_{i}$ is the normal state resistance of the $i$ th junction and $\Delta \mu_{i}$ is the difference in the chemical potential of the two droplets forming the $i$ th junction. The tunneling rates from the left can be similarly defined.

The difference in the chemical potential, $\Delta \mu_{i}$, can be written as

$\Delta \mu_{i}=e V_{i}-E_{\mathrm{c} i}$,

where $e V_{i}$ is the energy an electron gains when moving through a potential difference of $V_{i}$ and $E_{\mathrm{c} i}$ is the charging energy of the $i$ th junction. The charging voltage $V_{\mathrm{c} i}=E_{\mathrm{c} i} / e$ is the voltage that must be developed across the $i$ th junction before an electron will tunnel. The change in the chemical potential and hence, the charging energy, depends on the characteristic times of the system. We consider two limiting cases for $\Delta \mu_{i}$, that of "local" and "nonlocal" charging energy:

$\tau_{\mathrm{t}}<\tau_{\mathrm{r}}<\tau_{\mathrm{b}} \quad\left(\right.$ local); $\quad \tau_{\mathrm{r}}<\tau_{\mathrm{t}}<\tau_{\mathrm{b}} \quad$ (nonlocal);

where $\tau_{\mathrm{r}}$ is the response time of the system, $\tau_{\mathrm{r}}$ is the time the electron spends in the barrier during the tunneling process, and $\tau_{\mathrm{b}}$ is the time between tunneling events. For the local charging limit, the charge distribution throughout the array does not change until after the charge has tunneled. Hence, the charging energy of a junction is determined by the characteristics of that junction alone, and is the same as the charging energy for an isolated single junction, $E_{\mathrm{c} i}=e^{2} / 2 C_{i}$. In the nonlocal charging limit, the charge on every junction and coupling capacitor redistributes during the tunneling process, making the charging energy dependent on the entire system. To calculate $\Delta \mu_{i}$ in the nonlocal limit we assume that as the electron tunnels the junction voltages change linearly. Using this assumption we obtain

$\Delta \mu_{i}=-e \int_{\left\{N_{j}^{\mathrm{b}}\right\}}^{\left\{N_{j}^{\mathrm{a}}\right\}} \mathrm{d}\left\{N_{j}^{\prime}\right\} V_{i}\left(\left\{N_{j}^{\prime}\right\}\right)$, 
where $\left\{N_{j}^{\mathrm{b}}\right\}$ and $\left\{N_{j}^{\mathrm{a}}\right\}$ refer to the excess charge distribution before and after the tunneling event on the $i$ th junction. From eqs. (1)-(4) it is clear that the junction voltages are linear in $\left\{N_{j}\right\}$; therefore, eq. (9) becomes $\left.\Delta \mu_{i}=\left[V_{i}\left\{N_{j}^{\mathbf{a}}\right\}\right)+V_{i}\left(\left\{N_{j}^{\mathbf{b}}\right\}\right)\right] e / 2$. The charging energy is then $E_{\text {ci }}=\left[V_{i}\left(\left\{N_{j}^{\mathbf{b}}\right\}\right)-V_{i}\left(\left\{N_{j}^{\mathbf{a}}\right\}\right)\right] e / 2$. Since the voltage is redistributed during the tunneling process in the nonlocal limit, the nonlocal charging energy is less than the local charging energy. This is demonstrated in fig. 2 , where we show the local and nonlocal charging energies for a forty junction array. Note that in the nonlocal limit the charging energy tends to decrease at the boundaries. Also, as the ratio $C_{\mathrm{s}} / C$ is increased, the charging energy decreases. The larger coupling capacitances reduce the change in junction voltage due to tunneling by supplying or removing a larger amount of charge; therefore, the charging energy is reduced.

For simplicity, we will now consider the case of an array composed of identical junctions of capacitance $C$ and resistance $R$, and identical droplet-substrate coupling capacitances of $C_{\mathrm{s}}$. Before we discuss the results we note that from eqs. (1)-(4) the following is obtained:

$Q_{i}=\frac{\left(N_{i}-N_{i-1}\right) e+Q_{i-1}+Q_{i+1}}{2+C_{\mathrm{s}} / C}$

where $Q_{i}=V_{i} C$ is the charge on the $i$ th junction. (Note that this definition of $Q_{i}$ differs from that defined in a previous paper [5], where $Q_{i}$ was the total charge transferred to the $i$ th junction of the array.) Eq. (10) shows that the charge on the $i$ th junction is coupled to the charge on adjacent junctions. The strength of this coupling depends on the ratio $C_{\mathrm{s}} / C$. The coupling between the droplets is required in order to have correlated, solitonlike, transfer of charge. From eq. (10) it is clear that a zero droplet-substrate coupling $\left(C_{\mathrm{s}}=0\right)$ produces the largest coupling between the droplets. In this limit there is no soliton propagation since all the junctions are locked and the array acts as a single junction.

The time development of the junction voltages is determined by iterating the stochastic process (6) forward

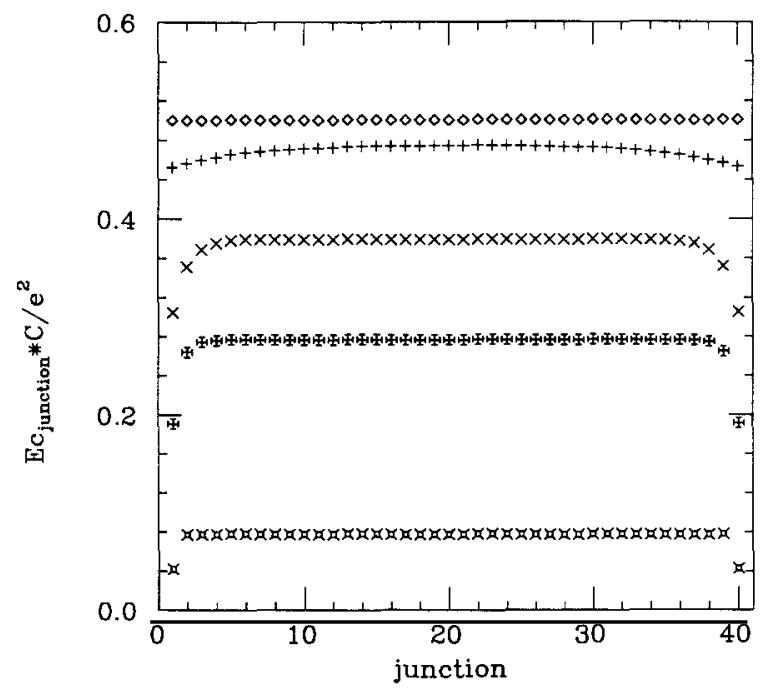

Fig. 2. Calculated charging energy as a function of junction number for a forty junction array formed from identical junctions of capacitance $C=0.01 \mathrm{fF}$ with identical droplet-substrate coupling. From top to bottom the curves correspond to local charging, nonlocal charging with $C_{\mathrm{s}} / C=0.01$, nonlocal charging with $C_{\mathrm{s}} / C=0.25$, nonlocal charging with $C_{\mathrm{s}} / C=1.0$, and nonlocal charging with $C_{\mathrm{s}} / C=10.0$.
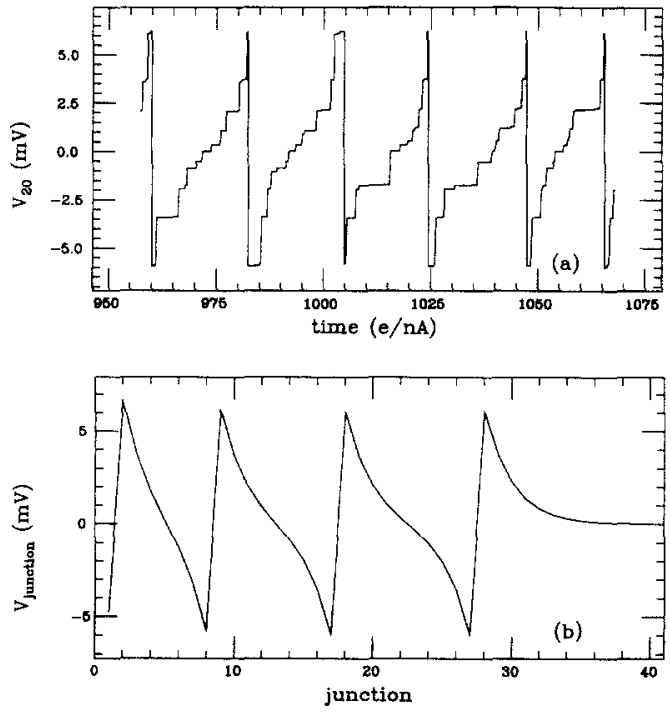

Fig. 3. (a) Calculated voltage dropped across junction number twenty in a forty junction array with junction coupling of $C_{\mathrm{s}} /$ $C=0.25$ in the nonlocal charging limit. The parameters are $C=0.01 \mathrm{fF}, R=100 \mathrm{k} \Omega, C_{\mathrm{s}}=0.0025 \mathrm{fF}, V=13.6 \mathrm{mV}$, and $T=0$ K. (b) Calculated junction voltages for the same system at time $=998.5 e / \mathrm{nA}$. 
in time. In fig. 3 we show that this model produces soliton-like modes of charge propagation. The voltage dropped across junction number twenty as a function of time in a forty junction array is shown in fig. 3a. Each step in the voltage corresponds to an electron tunneling somewhere in the array. The large jumps in voltage occur when an electron tunnels through junction number twenty. This time development at a low voltage clearly shows correlated charge transfer. An example of the voltage profile for the forty junction array is shown in fig. $3 \mathrm{~b}$. The jumps in the voltage occur at droplets where $N_{i}=-1$. The other droplets have $N_{i}=0$. One way to observe experimentally the solitons is to couple to one of the junctions, say with a scanning tunneling microscope, and observe the voltage as a function of time. One can also look at the current as a function of time which will show a periodicity that corresponds to the velocity of the solitons divided by the distance between the solitons. Another indirect method is to look for structure, produced by the correlated charge transfer, in the average current as a function of applied voltage for the array. We show below that this structure is in the form of steps in the $I-V$ characteristics.

Average current versus applied voltage characteristics of the system is determined by iterating the stochastic process of eq. (6) forward in time, for fixed applied voltage $V$, and averaging the charge transfer over a sufficiently long period of time. This averaging is performed for many values of the applied voltage to obtain a complete $I-V$ characteristic. In figs. 4-6 we show examples of numerically calculated $I-V$ characteristics of a forty junction array in the local and nonlocal charging limits. For the strong coupling limit $\left(C_{\mathrm{s}} / C \ll 1\right)$, the local and nonlocal curves are similar in shape because the charging energies in the two limits are very similar (see fig. 2). For $C_{\mathrm{s}}=0$ the charging energy in the nonlocal limit is independent of the junction number and is

$E_{\mathrm{c}}=\frac{e^{2}}{2 C} \frac{m}{m+1}$.

Since $e^{2} / 2 C$ is the charging energy in the local limit, similar $I-V$ characteristics are expected for strong coupling as the number of junctions gets large. Also, for $C_{\mathrm{s}}=0$, the voltage dropped across each junction is the same

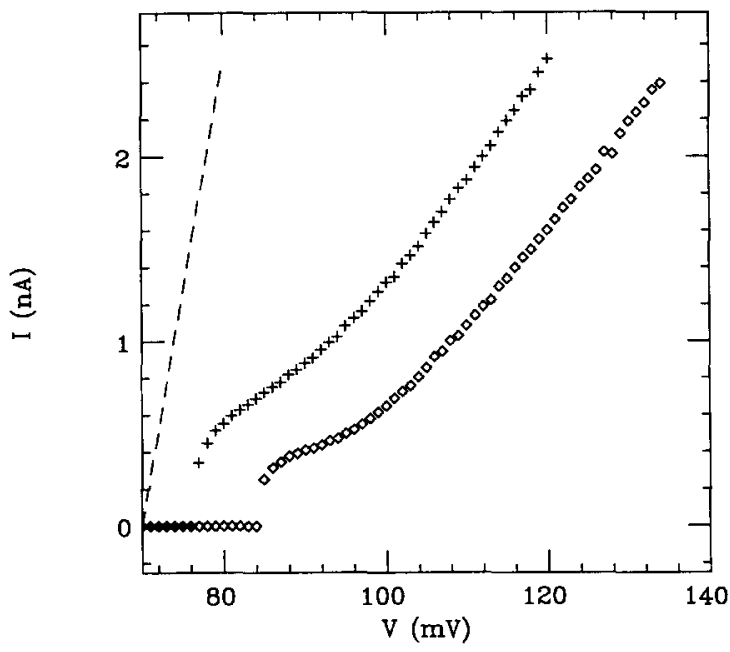

Fig. 4. Calculated $I-V$ characteristics for a forty junction array with strong junction coupling. The data shown with crosses corresponds to the nonlocal charging limit with $C=0.01 \mathrm{fF}, R=100$ $\mathrm{k} \Omega$, and $C_{\mathrm{s}}=0.0001 \mathrm{fF}$. The data shown with diamonds correspond to the local charging limit for the same parameters. The dashed line, shown for comparison, is $I=V(m+1) R$.

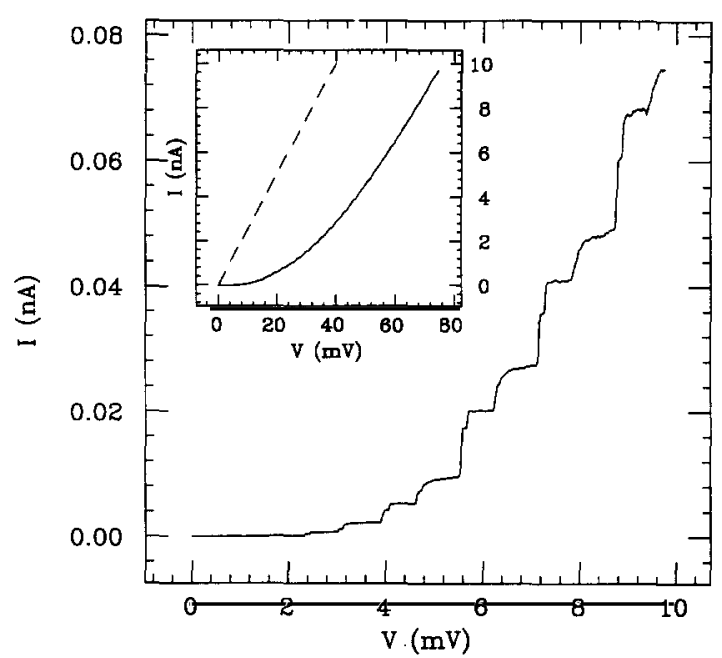

Fig. 5. Calculated $I-V$ characteristic for a forty junction array in the nonlocal charging limit with weak junction coupling. The parameters are $C=0.01 \mathrm{fF}, R=100 \mathrm{k} \Omega$, and $C_{\mathrm{s}}=0.1 \mathrm{fF}$. Inset: calculated $I-V$ characteristic for the same system at larger voltages. The dashed line is $I=V /(m+1) R$. 

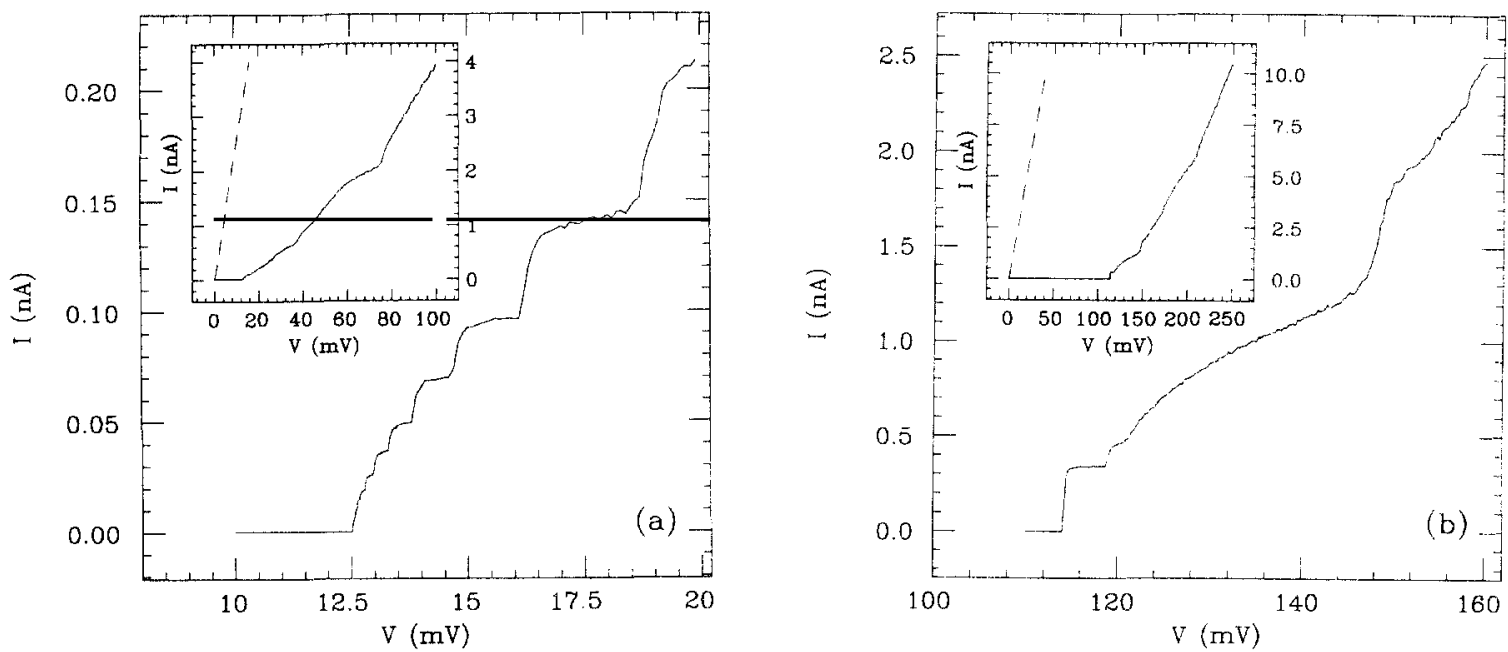

Fig. 6. Calculated $I-V$ characteristics for a forty junction array with a junction coupling of $C_{\mathrm{s}} / C=0.25$. The parameters are $C=0.01 \mathrm{fF}$. $R=100 \mathrm{k} \Omega$, and $C_{\mathrm{s}}=0.0025 \mathrm{fF}$. (a) Nonlocal charging limit. Inset: $I-V$ characteristic for the same system at larger voltages. The dashed line is $I=V /(m+1) R$. (b) Local charging limit. Inset: $I-V$ characteristic for the same system at larger voltages. The dashed line is $I=I /$ $(m+1 R$.

when $\left\{N_{j\}}\right\}=0$. The voltage at which charge could be transferred through the system is then $(m+1) e / 2 C$ in the local limit and $m e / 2 C$ in the nonlocal limit. The threshold voltages in fig. 4 are much less than these voltages because the substrate coupling causes more voltage to be dropped across the junctions closest to the voltage source. For this coupling, nonzero current occurs when the applied voltage is large enough to cause $V_{1}$ to be greater than $E_{\mathrm{cl}} / e$.

The case of weak coupling $\left(C_{\mathrm{s}} / C>1\right)$ in the nonlocal charging limit is shown in fig. 5. The $I-V$ characteristic resembles that of a single current driven junction with a charging energy approximately $m+1$ times the average charging energy of the array and a normal state resistance of $(m+1) R$. However, a closer look at small currents shows pronounced steps corresponding to different soliton modes. To understand the origin of these steps consider a three junction array. The first step from zero current corresponds to the system passing one soliton at a time across the array $\# 1$. (The system passes through the following states: $\left\{N_{1}, N_{2}\right\}=\{0,0\} \rightarrow\{-1,0\} \rightarrow\{0$, $-1\} \rightarrow\{0,0\} \rightarrow \ldots)$. When the applied voltage is increased, the system will be able to access additional paths. For example, the system may be able to go from $\{0,-1\}$ to $\{-1,-1\}$ then to $\{0,0\}$ in addition to going directly from $\{0,-1\}$ to $\{0,0\}$. The additional accessible state $\{-1,-1\}$ will produce a jump in the current. Similarly, in larger arrays, the system will pass through different paths and as the applied voltage gets larger additional paths will become accessible to the system, thus causing jumps in the current. We will discuss this in more detail elsewhere [12].

In fig. 6 we show $I-V$ characteristics for an intermediate coupling $\left(C_{s} / C=0.25\right)$. Again. note the structure at low currents. The threshold voltage for the local limit is much greater than that in the nonlocal limit due to the greater charging energies in the former limit. In this particular case, when $V \approx 12.5 \mathrm{mV}$ and $\left\{N_{j}\right\}=0$ the voltage on the first junction exceeds its charging voltage in the nonlocal limit, thus allowing charge to transfer. The charging voltages throughout the system are then of values low enough to allow charge to propagate through the system, near this value of the applied voltage. Whereas in the local limit, the applied voltage required to overcome the charging voltage on the first junction is approximately $21 \mathrm{mV}$ (for these parameters), which is

\#1 For certain junction parameters the first step from zero current will correspond to the system accessing more than the single path of passing a single soliton across the array. 
much less than the threshold voltage. In the local limit the junctions transfer charge at applied voltages lower than the threshold voltage, but this transfer of charge stops before charge is passed completely through the system. In other words, the array charges to a point when the junction voltages are less than their corresponding charging voltages. Nonzero current finally occurs when the charging of the system reaches across the entire array.

Recently, experiments have been done on one-dimensional arrays [10]. No steps of the type predicted here were reported in these experiments because the experiments were done in the strong coupling limit. (Their parameters correspond to $C_{\mathrm{s}} / C=0.05$.) We expect that by varying the ratio by a factor of five they should see structure in the $I-V$ characteristics due to the soliton-like charge propagation.

We have presented here a prediction of steps in the $I-V$ characteristics of a one-dimensional array of mesoscopic tunnel junctions due to soliton propagation. This prediction can be directly confirmed experimentally using existing systems $[9,10]$.

We have profited from many useful discussions with R.C. Jaklevic. We are thankful to P. Delsing, K.K. Likharev, L.S. Kuzmin, and T. Claeson for sending us ref. [10]. This research was partially funded by NSF Grant DMR 8608305, Grant DAAL 03-87-k-0007 from the Army Research Office, and the Presidential Initiative Fund at the University of Michigan. Computer simulations were done in part using time granted by the NSF Computer Center on the San Diego Super Computer. K.M. and M.A. are supported by fellowships from the Center for High Frequency Micro-electronics at the University of Michigan.

\section{References}

[1] E. Ben-Jacob and Y. Gefen, Phys. Lett. A 108 (1985) 289; E. Ben-Jacob, Y. Gefen, K. Mullen and Z. Schuss, in: SQUID 85, eds. H.D. Hahlbohm and H. Lübbig (De Gruyter, Berlin, 1985); E. Ben-Jacob, Y. Gefen, K. Mullen and Z. Schuss, Phys. Rev. B 37 (1988) 7400; K. Mullen, Y. Gefen and E. Ben-Jacob, Physica B 152 (1988) 172.

[2] D.V. Averin and K.K. Likharev, in: SQUID 85, eds. H.D. Hahlbohm and H. Lübbig (De Gruyter, Berlin, 1985); D.V. Averin and K.K. Likharev, J. Low Temp. Phys. 62 (1986) 345;

D.V. Averin and K.K. Likharev, in: Quantum effects in small disordered systems, eds. B.L. Altshuler, P.A. Lee and R.A. Webb, to be published.

[3] E. Ben-Jacob, F. Lamelas and K. Mullen, Josephson junctions: theory and applications, Lecture notes, University of Michigan, Ann Arbor (1988).

[4] K.K. Likharev, IBM J. Res. Dev. 32 (1988) 144.

[5] E. Ben-Jacob, K. Mullen and M. Amman, Phys. Lett. A 135 (1989) 390.

[6] K.K. Likharev, N.S. Bakhvalov, G.S. Kazacha and S.I. Serdyukova, IEEE Trans. Magn. 25 (1989) 1436.

[7] M. Büttiker and R. Landauer, Phys. Rev. Lett. 49 (1982) 1739.

[8] R. Wilkins, E. Ben-Jacob and R.C. Jaklevic, Phys. Rev. Lett. 63 (1989) 801.

[9] L.J. Geerligs, V.F. Anderegg, C.A. van der Jeugd, J.R. Romijn and J.E. Mooij, preprint.

[10] L.S. Kuzmin, P. Delsing, T. Claeson and K.K. Likharev, Phys. Rev. Lett. 62 (1989) 2539; P. Delsing, K.K. Likharev, L.S. Kuzmin and T. Claeson, preprint.

[11] K. Mullen, E. Ben-Jacob, R.C. Jaklevic and Z. Schuss, Phys. Rev. B 37 (1988) 98; M. Amman, K. Mullen and E. Ben-Jacob, J. Appl. Phys. 65 (1989) 339.

[12] M. Amman, E. Ben-Jacob and K. Mullen, unpublished. 